\title{
Matter creation in a nonsingular bouncing cosmology
}

\author{
Jerome Quintin, ${ }^{1, \text { f }}$ Yi-Fu Cai, ${ }^{1, \text { † }}$ and Robert H. Brandenberger ${ }^{1, \text { f }}$ \\ ${ }^{1}$ Department of Physics, McGill University, Montréal, Québec, H3A 2T8, Canada
}

\begin{abstract}
We examine reheating in the two-field matter bounce cosmology. In this model, the Universe evolves from a matter-dominated phase of contraction to an Ekpyrotic phase of contraction before the nonsingular bounce. The Ekpyrotic phase frees the model from unwanted anisotropies, but leaves the Universe cold and empty of particles after the bounce. For this reason, we explore two particle production mechanisms which take place during the course of the cosmological evolution: Parker particle production where the matter field couples only to gravity and particle creation via interactions between the matter field and the bounce field. Although we show that both mechanisms can produce particles in this model, we find that Parker particle production is sufficient to reheat the Universe to high temperatures. Thus there is a priori no need to add an interaction term to the Lagrangian of the model. Still, particle creation via interactions can contribute to the formation of matter and radiation, but only if the coupling between the fields is tuned to be large.
\end{abstract}

PACS numbers: $98.80 . \mathrm{Cq}$

\section{INTRODUCTION}

Reheating (see e.g. [1] for a recent review) is an integral part of inflationary cosmology. Without a mechanism to convert the energy residing in the inflaton field at the end of inflation into regular matter, inflation would produce a universe devoid of any regular matter and radiation, obviously a cosmological catastrophe. In a similar way, an early universe cosmology alternative to inflation must contain a mechanism which leads to regular matter and radiation at late times.

The matter production mechanisms will differ from model to model. In inflationary cosmology, it is a weak coupling between the inflaton field and the fields representing regular matter which leads to a parametric resonance ("preheating") instability in the equation of motion of these matter fields during the time after the end of inflation when the inflaton is oscillating about its ground state [2 5]. In "string gas cosmology" [6, it is the annihilation of string winding modes into string loops which automatically leads to the generation of matter and radiation at the end of the initial string phase (the "Hagedorn" phase - see e.g. 7] for recent reviews on string gas cosmology). In the "emergent Galileon cosmology" [8] it is a "defrosting" transition analogous to the resonant instability at the end of inflation which leads to the production of regular matter [9]. This mechanism also requires a coupling between regular matter fields and the Galileon condensate.

A nonsingular bouncing cosmology with a matterdominated initial phase of contraction (during which scales of cosmological interest exit the Hubble radius) is another alternative to cosmological inflation for producing the spectrum of cosmological fluctuations observed

\footnotetext{
*Electronic address: jquintin@physics.mcgill.ca

${ }^{\dagger}$ Electronic address: yifucai@physics.mcgill.ca

${ }^{\ddagger}$ Electronic address: rhb@hep.physics.mcgill.ca
}

today [10, 11. The nonsingularity of the bounce can be obtained by introducing new physics in the matter sector such as a quintom field [12] or a ghost condensate [13, or by modifying gravity at high scales, e.g. in the context of string theory [14], Horava-Lifshitz gravity [15], nonlocal gravity [16], or loop quantum cosmology [17]. If there is no additional phase of contraction during which the relative contribution of radiation to the total energy density decreases, then there is no need for a matter generation mechanism during and after the bounce since the original matter and radiation content of the Universe at early times during the contracting phase is preserved. However, it is precisely such bouncing models without a dilution mechanism during contraction which suffer from the "anisotropy problem" ["Belinsky-KhalatnikovLifshitz (BKL) instability" [18; the energy density in the anisotropies grows faster than the energy density in matter and radiation and destroys the homogeneous bounce]. To solve this anisotropy problem, a model with a phase of Ekpyrotic contraction was introduced [19, 20] and it was shown explicitly to be stable against anisotropies in 21] (see 22] for a review). However, during the period of Ekpyrotic contraction the regular matter and radiation become irrelevant analogously to what happens to preinflationary matter and radiation during inflationary expansion, and hence a mechanism is required to recreate matter and radiation during and after the bounce. This is the topic we address in the present study.

In this paper, we study gravitational matter field particle creation in the two-field Ekpyrotic matter bounce model of [20. This mechanism is called "Parker particle production" 23-25]. Due to the nontrivial dynamics of the matter mode functions in the evolving background space-time, we find that particles of matter fields can be efficiently generated. We evaluate the particle number at the moment of matter-Ekpyrotic equality, and at the beginning and end of the bouncing phase. Our results explicitly show that by the time of the end of the bouncing phase there is sufficient gravitational particle production, and that there is hence no need to introduce 
any preheating phase. This is very different from the situation in inflationary cosmology where gravitational particle production is negligible compared to the energy transfer via the preheating instability. We further show that even if we introduce a small coupling between the scalar field leading to Ekpyrotic contraction and the regular matter fields, then parametric resonance is in fact negligible.

The paper is organized as follows. In Sec. II, we briefly review the model of two-field matter bounce cosmology proposed in Ref. 20]. In Sec. III, we study gravitational particle creation of a regular scalar matter field $\psi$ by tracking the background evolution. In particular, we present the quantization process of the $\psi$ particles in the matter contraction, Ekpyrotic contraction, and the bouncing phases, respectively. Afterwards, we compute the Bogoliubov coefficients of the $\psi$ particles by following the background evolution, and we determine the magnitude of the energy density in the produced particles. To double check the validity of the analyses of Sec. III, we analyze in Sec. IV the same setup by computing the backreaction of the $\psi$ fluctuations on the energy-momentum tensor. Then in Sec. V, we turn on an interaction term coupling the matter field to the field yielding the Ekpyrotic contraction, and we investigate particle production through direct interactions. We conclude with a discussion in Sec. VI. Throughout the paper we take the sign of the metric to be $(+,-,-,-)$ and define the reduced Planck mass by $M_{p}=1 / \sqrt{8 \pi G}$.

\section{THE TWO-FIELD MATTER BOUNCE MODEL}

The two-field matter bounce 20] is a simple toy model which yields regular matter and can give both an Ekpyrotic contraction and a nonsingular bounce. In this section, we briefly review the dynamics of this nonsingular bounce model.

Following [20], we consider the Lagrangian of the model to be

$$
\mathcal{L}=K(\phi, X)+G(X) \square \phi+P(\psi, Y)
$$

where $\phi$ is the scalar field responsible for both the Ekpyrotic phase of contraction and the bounce, and $\psi$ is a second scalar field representing the regular matter component responsible for the initial matter contraction phase.

For the scalar field $\phi$, we introduce a $K$-essence-type term $K$ which is a function of $\phi$ and its kinetic term [26]

$$
X \equiv \frac{1}{2} g^{\mu \nu} \partial_{\mu} \phi \partial_{\nu} \phi
$$

and a Horndeski-type [27] term $G \square \phi$ with

$$
\square \equiv g^{\mu \nu} \nabla_{\mu} \nabla_{\nu}
$$

being the standard d'Alembertian operator. When the Universe is far away from the bounce, the Lagrangian of $\phi$ takes the canonical form which is the sum of a regular kinetic term $X$ and a potential $V(\phi)$. More specifically, we choose

$$
K(\phi, X)=M_{p}^{2}[1-g(\phi)] X+\beta X^{2}-V(\phi)
$$

and

$$
G(\phi, X)=\gamma X
$$

with $\beta$ and $\gamma$ being two positive-definite constants, and

$$
g(\phi)=\frac{2 g_{0}}{e^{-\sqrt{2 / p} \phi}+e^{b_{g} \sqrt{2 / p} \phi}},
$$

where $g_{0}>1, p$, and $b_{g}$ are further positive constants. If the potential is chosen to be a negatively valued exponential function, one can get a phase of Ekpyrotic contraction 28] which will dilute the unwanted anisotropies [29]. The choice made in 20] for the potential was

$$
V(\phi)=\frac{2 V_{0}}{e^{-\sqrt{2 / q} \phi}+e^{b_{V} \sqrt{2 / q} \phi}},
$$

where $V_{0}, q$, and $b_{V}$ are more positive constants. As a result, this type of model can avoid the BKL instability. The initial conditions for $\phi$ will be taken to be a large negative value with small time derivative. In this case, $\phi$ will contribute negligibly to the energy and momentum of the background. As $\phi$ increases, it will eventually reach a value for which $g(\phi)>1$ and hence a ghost condensate 30] will form for a short while. This gives rise to a nonsingular bounce via the violation of the null energy condition.

Now we consider the dynamics of the massive scalar field $\psi$ which represents the regular matter component. We take the Lagrangian to be of canonical form as

$$
P(\psi, Y)=Y-\frac{1}{2} m^{2} \psi^{2},
$$

with $Y$ being defined as the kinetic term

$$
Y \equiv \frac{1}{2} g^{\mu \nu} \partial_{\mu} \psi \partial_{\nu} \psi
$$

and $m$ being the mass of the scalar. When $\psi$ oscillates around its vacuum $\langle\psi\rangle=0$, its contribution to the energy-momentum tensor is that of a pressureless fluid. If it dominates the total energy-momentum tensor, then it leads to a matter-dominated evolution.

Let us take a universe filled with the above two fields and consider a contracting universe with initial conditions in which the contribution of the Ekpyrotic field to the energy-momentum tensor is negligible since $\phi$ is initialized to have large negative value and small velocity. Hence, the Universe will undergo a phase of matter-dominated contraction with an effective background equation of state $w=0$. Since the energy density in the Ekpyrotic field increases faster than that in $\psi$, the Universe will at some point enter a period of Ekpyrotic 


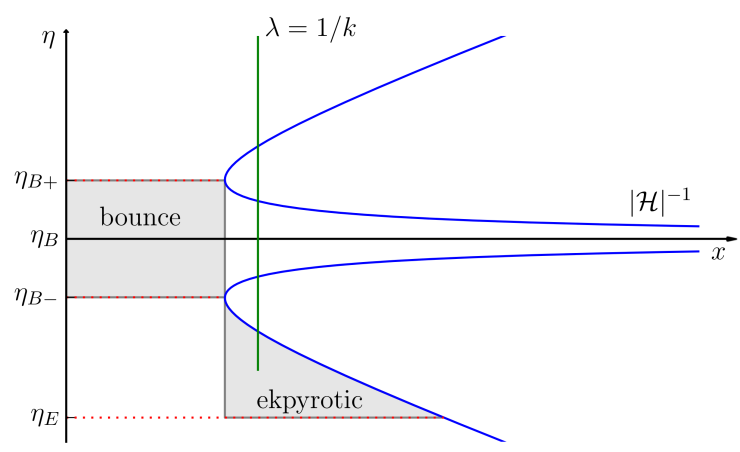

FIG. 1: Space-time sketch in the two-field matter bounce scenario. The comoving length and conformal time are labeled by $x$ and $\eta$, respectively. The solid blue curve shows $\mathcal{H}^{-1}$, the comoving Hubble radius. The different phase transition times are labeled on the vertical axis. The shaded areas show the regions of integration for the respective phases (the Ekpyrotic and bounce phases are shown). The comoving wavelengths of the fluctuating modes that we integrate originate in these shaded areas. The green line shows the comoving wavelength of such a fluctuating mode that forms in the Ekpyrotic phase of contraction. We see that this mode reenters the Hubble horizon at later times, after $\eta_{B+}$.

contraction driven by the scalar field $\phi$, with $w$ evolving to $w \gg 1$. Afterwards, the Universe experiences a nonsingular bounce due to the emergence of the ghost condensate phase. The ghost condensate phase ends naturally once $\phi$ exceeds a critical positive value. At that point traditional cosmological thermal expansion takes over. The space-time diagram of our nonsingular bounce cosmology is sketched in Fig. 1 in which the vertical axis denotes time, and the horizontal axis denotes a comoving space coordinate. The bounce point is taken to be $t=0$. The wavelength of one mode is depicted (the vertical line), and the Hubble radius is shown. It decreases in comoving coordinates during the contraction phase and then increases again in the expanding phase.

Since in the two-field matter bounce model we use the Ekpyrotic phase of contraction to dilute the undesired anisotropies, this phase also washes out the primordial matter particles. Therefore, one must be concerned that the Universe might be empty of regular matter and radiation after the bounce. As discussed in the Introduction, a similar issue arises within the framework of inflationary cosmology. In the case of inflation, in order to have efficient production of regular matter after the phase of exponential expansion, one usually introduces an explicit coupling term between the field $\phi$ which yields inflation and regular matter fields. In this case, already at the level of quantum field perturbation theory one expects the production of matter particles [31, 32] after inflation has ended, i.e. in a separate phase called the "reheating period." However, it was observed in 2] (see also [33]) that the initial transfer of energy to matter fields is dominated by a parametric resonance instability. This "preheating period" was further analyzed in 3 5].
The question now arises as to whether it is necessary to introduce an analogous reheating phase in a nonsingular matter bounce scenario in order to explain the origin of regular matter and radiation. It is obviously possible to introduce such a mechanism, and this was done within the framework of nonsingular bouncing cosmologies in order to enhance the amplitude of the scalar cosmological perturbations relative to the tensor modes [34. However, since the scalar modes in fact experience a substantial growth in amplitude during the bouncing phase (greater than that of the tensor modes) [20] (see also [35] for earlier work on fluctuations in a nonsingular bouncing cosmology), it is not necessary to invoke bounce preheating to boost the scalar fluctuations, and it would be more elegant not to do so since the bounce preheating scenario requires additional couplings (like inflationary reheating does).

In this present work, we analyze matter production in the two-field matter bounce model and show that there is no need to introduce preheating. Gravitational particle production is in fact sufficiently effective. Specifically, we consider the production of $\psi$ particles in our cosmological background. We are interested in particles with momenta which are large (compared to the Hubble rate in the postbounce universe) rather than the large wavelength fluctuations which contribute to cosmological perturbations.

\section{GRAVITATIONAL PARTICLE PRODUCTION THROUGHOUT THE COSMIC EVOLUTION}

In an expanding universe the concept of the vacuum state of a quantum field is not well defined. In Minkowski space-time quantum field theory we can expand the field in creation and annihilation operators which are the operator coefficients of the positive and negative frequency modes, respectively. The vacuum state is then the state which is annihilated by all of the annihilation operators. In curved space-time there is no global definition of positive and negative frequency modes. A mode which to an initial observer looks pure positive frequency will look like a combination of positive and negative frequency modes for a later time observer. The late time observer thus sees the state as containing particles. This is the idea of Parker particle production (see [23 25]).

In the following, we will study Parker production of regular matter (i.e. $\psi$ ) particles in our nonsingular bouncing cosmology. Particle production is particularly efficient for modes which have a wavelength larger than the Hubble radius. For such modes, the canonical field variable is frozen out and squeezed. If the squeezed mode function which was initially positive frequency is expanded at late times into plane wave modes, then it obtains a large effective negative frequency component which corresponds to the production of potential $\chi$ particles (the use of the word "potential" indicates that we 
can only speak of true particles once the modes have reentered the Hubble radius and started to oscillate again). The mixing between the initial positive frequency modes and the late time modes is given by Bogoliubov coefficients, and their values give us the number of produced particles (see 36] for a textbook treatment of quantum field theory in curved space-time).

We will focus on modes which have wavelengths which already at early times (the time when we want to compute the density of regular matter particles) allow an interpretation of the field excitations as particles (i.e. short wavelengths). We set up these modes in their initial vacuum state and compute the Bogoliubov coefficients at later times in order to determine the number of produced particles. Integrating over Fourier modes then lets us calculate the energy density in the produced matter particles.

To begin, let us recall the basic equations we will use. Consider a homogeneous, isotropic and spatially flat FLRW universe. The corresponding background dynamics is governed by the energy density and the pressure of the different scalar fields (with energy densities are pressures denoted by $\rho$ and $P$, respectively) through the Friedmann equations, which are given by

$$
\begin{aligned}
H^{2} & =\frac{1}{3 M_{p}^{2}}\left(\rho_{\phi}+\rho_{\psi}\right) \\
\dot{H} & =-\frac{1}{2 M_{p}^{2}}\left(\rho_{\phi}+P_{\phi}+\rho_{\psi}+P_{\psi}\right),
\end{aligned}
$$

where $H \equiv \dot{a} / a$ is the Hubble rate of the Universe and the dot denotes the derivative with respect to the cosmic time. In addition, the scalar fields satisfy the continuity equation so that covariant energy conservation is guaranteed. The continuity equations can be derived by varying the Lagrangian with respect to the scalar fields. Namely, if we focus on the matter field $\psi$, the resulting equation of motion is

$$
\left(\square+m^{2}\right) \psi=0,
$$

which is the Klein-Gordon equation.

At the background level, the matter field $\psi$ is only a function of cosmic time. However, in order to study the quantum dynamics of its particle generation, we include the gradient term and hence write down the equation of motion of the field as follows,

$$
\ddot{\psi}+3 H(t) \dot{\psi}-\frac{\nabla^{2}}{a^{2}} \psi+m^{2} \psi=0,
$$

with $\nabla^{2} \equiv g^{i j} \nabla_{i} \nabla_{j}$. Note that, in the current model we have ignored possible interactions between $\psi$ and other fields. For now, we focus on particle production of the field $\psi$ only under gravitational effects. This assumption is in agreement with our starting point that all fields are weakly coupled.

For convenience, let us work in conformal time, which is related to the cosmic time via $d \eta=a^{-1}(t) d t$. As is well known, the matter equation simplifies if written in terms of the rescaled field $\chi$ defined via

$$
\chi(\eta, \mathbf{x})=a(\eta) \psi(\eta, \mathbf{x}) .
$$

The equation of motion for $\psi$ is then easy to translate into an equation of motion for $\chi$. Since the equation of motion is linear, each Fourier mode evolves independently. It is thus convenient to consider the Fourier transformation on the field $\chi$,

$$
\chi(\eta, \mathbf{x})=\int \frac{d^{3} k}{(2 \pi)^{3 / 2}} \chi_{k}(\eta) e^{i \mathbf{k} \cdot \mathbf{x}},
$$

and then track the dynamics of each Fourier mode along with the evolution of the background universe. The equation of motion for a Fourier mode of $\chi$ becomes

$$
\chi_{k}^{\prime \prime}+\omega_{k}^{2}(\eta) \chi_{k}=0
$$

with

$$
\omega_{k}^{2} \equiv k^{2}+a^{2} m^{2}-\frac{a^{\prime \prime}}{a},
$$

where primes denote derivatives with respect to conformal time. It is the final term in (16) which is responsible for the squeezing of the modes.

\section{A. Dynamics of the variable $\chi_{k}$}

We will solve the equation of motion (15) phase by phase throughout the cosmological evolution. Specifically, we divide the time line into four separate phases: the initial matter-dominated phase of contraction, the Ekpyrotic phase of contraction, the nonsingular bouncing phase, and finally the fast roll phase of expansion. In each of the phases, different approximations are applicable.

\section{Matter contraction}

We first study the dynamics of $\chi_{k}$ in the matterdominated phase of contraction. In this stage, the background scale factor evolves as

$$
a(\eta) \simeq c_{E}\left(\eta-\tilde{\eta}_{E}\right)^{2}, \text { with } c_{E} \equiv \frac{a_{E}}{\left(\eta_{E}-\tilde{\eta}_{E}\right)^{2}},
$$

where we introduce $a_{E} \equiv a\left(\eta_{E}\right)$ as the value of the scale factor at the end of this phase (and correspondingly, at the beginning of the Ekpyrotic phase). The coefficient $\tilde{\eta}_{E} \equiv \eta_{E}-2 / \mathcal{H}_{E}$ would correspond to the conformal time at the Big Crunch singularity if the matter phase of contraction lasted forever. Its inclusion is very useful in regularizing the detailed computation. Additionally, we have introduced the conformal Hubble rate $\mathcal{H} \equiv a^{\prime} / a$. 
Applying the relation (17) to the definition of the effective frequency square $(16)$, one can derive

$$
\omega_{k}^{m}(\eta)^{2}=k^{2}+c_{E}^{2} m^{2}\left(\eta-\tilde{\eta}_{E}\right)^{4}-\frac{2}{\left(\eta-\tilde{\eta}_{E}\right)^{2}},
$$

where the superscript " $m$ " denotes the phase of matter contraction. Then, by solving the key equation of motion (15), one can determine the solution of $\chi_{k}$. At early times, i.e. for $\eta \ll \eta_{E}$, it is obvious that the first two terms in Eq. (18) for the effective frequency are dominant. Accordingly, the solution for the variable $\chi_{k}$ is approximately the plane wave solution. Requiring vacuum initial conditions, we find the solution to be given by

$$
\chi_{k}^{m}(\eta) \simeq \frac{e^{-i \omega_{k}^{m}\left(\eta-\tilde{\eta}_{E}\right)}}{\sqrt{2 \omega_{k}^{m}}}
$$

\section{Ekpyrotic contraction}

In the two-field matter bounce model, the potential of the scalar field $\phi$ is negative and of exponential form. This kind of potential can drive $\phi$ into the attractor trajectory for which the effective equation of state is larger than unity 28]. As the Universe contracts, the energy density of the scalar field $\phi$ catches up with that of the matter field $\psi$, and subsequently, a period of Ekpyrotic contraction begins. During this period, the background scale factor can be expressed as a function of the conformal time in the following approximate form

$$
a(\eta) \simeq c_{B-}\left(\eta-\tilde{\eta}_{B-}\right)^{\frac{q}{1-q}}, \quad \text { with } c_{B-} \equiv \frac{a_{B-}}{\left(\eta-\tilde{\eta}_{B-}\right)^{\frac{q}{1-q}}}
$$

and where $a_{B-} \equiv a\left(\eta_{B-}\right)$ is the scale factor at the end of Ekpyrotic phase of contraction (and equivalently, at the beginning of the bounce phase). Similar to the treatment of the phase of matter contraction, we have introduced $\tilde{\eta}_{B-} \equiv \eta_{B-}-\frac{q}{(1-q) \mathcal{H}_{B-}}$ in the above expression. Additionally, the parameter $q$ is associated with the background dynamics and is required to be much less than unity so that the Ekpyrotic phase is achieved.

Making use of (20), one can easily obtain the squeezing term in the equation of motion for $\chi_{k}$ :

$$
\frac{a^{\prime \prime}}{a}=-\frac{q(1-2 q)}{(1-q)^{2}} \frac{1}{\left(\eta-\tilde{\eta}_{B-}\right)^{2}} .
$$

The mass term $a^{2} m^{2}$ is subdominant since the scale factor has decreased to a small value and the energy scale of the Universe has become higher than the mass scale $m$ after matter contraction. Therefore, the dominant part of Eq. (16) is given by

$$
\omega_{k}^{c}(\eta)^{2}=k^{2}+\frac{q(1-2 q)}{(1-q)^{2}} \frac{1}{\left(\eta-\tilde{\eta}_{B-}\right)^{2}},
$$

where the superscript " $c$ " denotes the phase of Ekpyrotic contraction.

Substituting the expression 22 into the equation of motion (15), one can solve the differential equation and find the explicit general solution to be

$$
\begin{aligned}
\chi_{k}^{c}(\eta)= & \sqrt{\eta-\tilde{\eta}_{B-}}\left\{C_{1}(k) J_{\nu_{c}}\left[k\left(\eta-\tilde{\eta}_{B-}\right)\right]\right. \\
& \left.+C_{2}(k) Y_{\nu_{c}}\left[k\left(\eta-\tilde{\eta}_{B-}\right)\right]\right\}
\end{aligned}
$$

where $J_{\nu_{c}}$ and $Y_{\nu_{c}}$ are the two linearly independent Bessel functions of the first and second kind, respectively. They have the same index

$$
\nu_{c}=\frac{1-3 q}{2(1-q)} \sim \frac{1}{2} .
$$

For small values of the argument, i.e. for $\left|k\left(\eta-\tilde{\eta}_{B-}\right)\right|<1$, the scalings of the solutions are

$$
\chi_{k} \sim\left(\eta-\tilde{\eta}_{B-}\right)^{q} \text { and } \chi_{k} \sim\left(\eta-\tilde{\eta}_{B-}\right)^{1-q},
$$

which translates to

$$
\psi_{k} \sim \text { const and } \psi_{k} \sim\left(\eta-\tilde{\eta}_{B-}\right)^{1-2 q} .
$$

We see that there is one (almost) constant mode, while the second mode decays.

Modes in the large $k$ limit with $\left|k\left(\eta-\tilde{\eta}_{B-}\right)\right| \gg 1$ are still inside the Hubble radius and keep the oscillating behavior. In this case, the solution can be simplified into the following asymptotic form

$$
\chi_{k}^{c}(\eta) \simeq \frac{1}{\sqrt{2 k}}\left[\bar{C}_{1}(k) e^{i k\left(\eta-\tilde{\eta}_{B-}\right)}+\bar{C}_{2}(k) e^{-i k\left(\eta-\tilde{\eta}_{B-}\right)}\right]
$$

with

$$
\bar{C}_{1}=\frac{2 i+q \pi}{2 \sqrt{\pi}}\left(C_{1}+i C_{2}\right), \quad \bar{C}_{2}=\frac{-2 i+q \pi}{2 \sqrt{\pi}}\left(C_{1}-i C_{2}\right),
$$

up to leading order. One can see that the $\bar{C}_{1}$ and $\bar{C}_{2}$ terms represent the negative and positive frequency modes, respectively. If $\bar{C}_{1}$ is vanishing, the solution to $\chi_{k}$ is identified as the vacuum fluctuation. However, if $\bar{C}_{1}$ is not zero, there will be particle production from the vacuum state.

\section{Bouncing phase}

The phase of Ekpyrotic contraction stops when the scalar field $\phi$ evolves into the ghost condensate state. Afterwards, the Universe experiences a nonsingular bouncing phase. To quantitatively characterize this phase, it is useful to approximately parametrize the Hubble parameter as a linear function of the cosmic time

$$
H(t)=\Upsilon t
$$


where $\Upsilon$ is a positive coefficient that determines how fast the bounce takes place. We denote the beginning and the end of the bounce phase by $t_{B-}$ and $t_{B+}$, respectively.

Using conformal time, we can solve for the scale factor near the bounce,

$$
a(\eta)=a_{B}+\frac{1}{2} a_{B}^{3} \Upsilon \eta^{2}+\mathcal{O}\left(\Upsilon^{2} \eta^{4}\right),
$$

under the fast bounce assumption (i.e. $\Upsilon \eta^{2}<1$ during the bouncing phase). We note that it is convenient to set the bounce point at $t_{B}=\eta_{B}=0$. We can then write down, up to leading order, the effective frequency square in the bounce phase,

$$
\omega_{k}^{b}(\eta)^{2}=k^{2}+a_{B}^{2}\left(m^{2}-\Upsilon\right),
$$

where the superscript " $b$ " denotes the bounce phase. In this case, the solution can be simply expressed as follows,

$$
\chi_{k}^{b}(\eta) \simeq \frac{1}{\sqrt{2 \omega_{k}^{b}}}\left[D_{1}(k) e^{i k \eta}+D_{2}(k) e^{-i k \eta}\right],
$$

again for the large $k$ modes.

\section{Fast roll expansion}

After the bounce, in the absence of backreaction of the fluctuations produced before and during the bounce, the Universe would experience a period of fast roll expansion with an effective equation of state $w=1$. The scale factor then evolves as

$$
a(t)=c_{B+}\left(\eta-\tilde{\eta}_{B+}\right)^{\frac{1}{2}}, \quad \text { with } c_{B+} \equiv \frac{a_{B+}}{\left(\eta_{B+}-\tilde{\eta}_{B+}\right)^{\frac{1}{2}}} .
$$

Analogously as before, $a_{B+}$ is the value of the scale factor after the bounce phase (and, correspondingly, at the beginning of this phase of fast roll expansion). Also, we define $\tilde{\eta}_{B+} \equiv \eta_{B+}-\frac{1}{2 \mathcal{H}_{B+}}$, which would correspond to the conformal time at the big bang if there were no bounce. The above equation yields

$$
\frac{a^{\prime \prime}}{a}=-\frac{1}{4\left(\eta-\tilde{\eta}_{B+}\right)^{2}},
$$

in the phase of fast roll expansion.

One can substitute the scale factor (33) and the derived relation (34) into the expression (16) to determine the effective frequency square in the stage of fast roll expansion. We find that the mass term is negligible when the Hubble parameter of the Universe $H$ is larger than the mass of the matter field $m$. As a result, we obtain the dominant part of the effective frequency squared:

$$
\omega_{k}^{e}(\eta)^{2}=k^{2}+\frac{1}{4\left(\eta-\tilde{\eta}_{B+}\right)^{2}} .
$$

As usual, the superscript " $e$ " denotes the phase of fast roll expansion.

Making use of (35), the equation of motion (15) yields the following solution,

$$
\begin{aligned}
\chi_{k}^{e}(\eta)= & \sqrt{\eta-\tilde{\eta}_{B+}}\left\{E_{1}(k) J_{0}\left[k\left(\eta-\tilde{\eta}_{B-}\right)\right]\right. \\
& \left.+E_{2}(k) Y_{0}\left[k\left(\eta-\tilde{\eta}_{B-}\right)\right]\right\},
\end{aligned}
$$

where $E_{1}$ and $E_{2}$ are two coefficients to be determined later. Again, we focus on the regime of large $k$ modes $\left(\left|k\left(\eta-\tilde{\eta}_{B+}\right)\right| \gg 1\right)$ and can get the simplified form,

$$
\chi_{k}^{e}(\eta) \simeq \frac{1}{\sqrt{2 k}}\left[\bar{E}_{1}(k) e^{i k\left(\eta-\tilde{\eta}_{B+}\right)}+\bar{E}_{2}(k) e^{-i k\left(\eta-\tilde{\eta}_{B+}\right)}\right],
$$

with

$$
\begin{aligned}
& \bar{E}_{1}=\frac{2 i+\pi / 3}{2 \sqrt{\pi}}\left(E_{1}+i E_{2}\right), \\
& \bar{E}_{2}=\frac{-2 i+\pi / 3}{2 \sqrt{\pi}}\left(E_{1}-i E_{2}\right),
\end{aligned}
$$

up to leading order.

\section{B. Particle production}

We are interested in whether particles of the matter field have been generated in the process of the evolution during the above different phases. As is conventionally done in studying quantum field theory in curved spacetime, we work in the Heisenberg representation in which the operators carry the time dependence.

To study particle production, it is important to express the solution of the field variable $\chi_{k}$ at a given time $\eta$ as a sum of local positive and negative frequency modes as follows,

$$
\chi_{k}=\frac{1}{\sqrt{2 \omega_{k}}}\left[\alpha_{k} e^{-i \int^{\eta} \omega_{k} d \tilde{\eta}}+\beta_{k} e^{i \int^{\eta} \omega_{k} d \tilde{\eta}}\right],
$$

where the coefficients $\alpha_{k}$ and $\beta_{k}$ are the so-called Bogoliubov coefficients. If the mode functions evolve adiabatically, then the Bogoliubov coefficients are time independent and no particles are produced.

Following the standard approach of canonical quantization, one can define the conjugate momentum $\Pi_{k}$ for the field variable through

$$
\Pi_{k} \equiv \frac{\delta \mathcal{S}}{\delta \chi_{k}}=\chi_{k}^{\prime} .
$$

Note that the general solutions to the field variable in different phases have been obtained in the expressions (19), 27), 32, and (37), respectively. In each phase, there are two modes. Given initial conditions at the beginning of the dynamics, the actual solution in each phase is the combination of the two fundamental solutions in that phase which is obtained by demanding the solutions 
to the variable $\chi$ and its conjugate momentum (and hence its time derivative) to be continuous across the transition between the previous phase and the one under consideration. Given the solution in any given phase, we can then determine the Bogoliubov coefficients. In doing this, we interpret the "amount" of negative frequency modes that are created during the entire evolution as the particle production.

We wish to consider the evolution of the vacuum state of $\chi_{k}^{\mathrm{vac}}(\eta)$ defined at some initial time $\eta_{i}$. First of all, we expand the field into combinations $\chi_{k}^{\mathrm{vac}}$ and $\chi_{k}^{\mathrm{vac} *}$ of the two fundamental solutions of the classical mode equation which are positive and negative frequency at $\eta_{i}$, respectively. After quantization, the coefficients $a_{\mathbf{k}}$ and $a_{\mathbf{k}}^{\dagger}$ become operators obeying the canonical commutation relations, which in turn implies that the $\chi$ field and its conjugate momentum $\Pi$ are canonically conjugate operators. It is then possible to write the mode expansion of the $\chi$ field as

$$
\chi(x)=\int \frac{d^{3} k}{(2 \pi)^{3 / 2}}\left(a_{\mathbf{k}} \chi_{k}^{\mathrm{vac}} e^{i \mathbf{k} \cdot \mathbf{x}}+a_{\mathbf{k}}^{\dagger} \chi_{k}^{\mathrm{vac} *} e^{-i \mathbf{k} \cdot \mathbf{x}}\right),
$$

where $a_{\mathbf{k}}^{\dagger}$ and $a_{\mathbf{k}}$ represent the creation and annihilation operators, respectively. The time dependence of the field operator is manifested by the time dependence of the classical mode functions.

At a later time $\eta_{\text {new }}$ we must expand the field operator $\chi$ in a new basis of mode functions which are locally positive and negative frequency at the new time. Let us denote the positive frequency mode at time $\eta_{\text {new }}$ by $\chi_{k}^{\text {new }}(\eta)$. The field $\chi$ can also be expanded in terms of the new basis modes

$$
\chi(x)=\int \frac{d^{3} k}{(2 \pi)^{3 / 2}}\left(b_{\mathbf{k}} \chi_{k}^{\text {new }} e^{i \mathbf{k} \cdot \mathbf{x}}+b_{\mathbf{k}}^{\dagger} \chi_{k}^{\text {new } *} e^{-i \mathbf{k} \cdot \mathbf{x}}\right),
$$

where $b_{\mathbf{k}}^{\dagger}$ and $b_{\mathbf{k}}$ are new creation and annihilation operators. Since $\chi_{k}^{\mathrm{vac}}$ and $\chi_{k}^{\text {new }}$ form complete sets of mode solutions at early and late times, it is possible to relate one set of modes to the other via

$$
\chi_{k}^{\text {new }}=\alpha_{k} \chi_{k}^{\mathrm{vac}}+\beta_{k} \chi_{k}^{\mathrm{vac} *},
$$

where the coefficients are the famous Bogoliubov coefficients. From this, it is straightforward to derive that the expectation value of the vacuum number operator evaluated after the phase transition is equal to

$$
n_{k}=\left|\beta_{k}\right|^{2} \text {. }
$$

This is the result that we will exploit throughout this work and interpret as the particle number in mode $k$ which has been produced between the initial time and the time $\eta_{\text {new }}$.

\section{Particle number at the beginning of the Ekpyrotic phase}

We start our modes in their vacuum state during the matter phase of contraction. At the time $t_{E}$, the equation of state transits into an Ekpyrotic one. As we found in the previous subsections, the fluctuating mode solutions $\chi_{k}$ in the matter contracting phase and Ekpyrotic contraction period are given by 19 and (27), respectively. To relate the two in the limit of small $q$, we can apply the matching condition by requiring the solutions and their conjugate momenta to be continuous at the transition point. This then yields up to leading order the following relation:

$$
\begin{aligned}
\beta_{k} \simeq & \frac{e^{-i \omega_{k}^{c}\left(\eta_{E}\right) \eta_{E}-i \omega_{k}^{m}\left(\eta_{E}\right)\left(\eta_{E}-\tilde{\eta}_{E}\right)}}{8 \sqrt{3} k^{2}} \\
& \times\left(\frac{2 q\left(\tilde{\eta}_{B-}+\eta_{E}\right)}{\left(\tilde{\eta}_{B-}-\eta_{E}\right)^{3}}-10 m^{2} c_{E}^{2}\left(\eta_{E}-\tilde{\eta}_{E}\right)^{4}\right),
\end{aligned}
$$

for fluctuation modes with comoving wave number $k$ larger than the comoving Hubble parameter $\mathcal{H}_{E}$. The number of particles that is then produced is

$$
\begin{aligned}
n_{k}=\left|\beta_{k}\right|^{2} \simeq & \frac{1}{192 k^{4}}\left[100 m^{4} c_{E}^{4}\left(\eta_{E}-\tilde{\eta}_{E}\right)^{8}\right. \\
& \left.-\frac{40 q m^{2} c_{E}^{2}\left(\eta_{E}-\tilde{\eta}_{E}\right)^{4}\left(\tilde{\eta}_{B-}+\eta_{E}\right)}{\left(\tilde{\eta}_{B-}-\eta_{E}\right)^{3}}\right] .
\end{aligned}
$$

To evaluate the contribution of these new particles to the background evolution, one should compute the energy density of these particles and compare it to the background energy density. Let us take for granted the fact that the energy density of the particles that have been produced is given by

$$
\rho_{\chi}=\frac{1}{(2 \pi)^{3} a^{4}} \int d^{3} k n_{k} \omega_{k} \simeq \frac{1}{2 \pi^{2} a^{4}} \int d k k^{3} n_{k},
$$

in the limit where $\omega_{k} \simeq k$. We will justify that this is the form that the energy density of particle creation should take in Sec. IV. However, similar to the vacuum energy density, it is easy to realize that this integral diverges, both in the ultraviolet (UV) and in the infrared (IR). For this reason, it is necessary to introduce some cutoffs. First, all of the above analysis has been done for modes on sub-Hubble scales at the initial time. Thus, the comoving scale corresponding to the initial Hubble radius yields the natural IR cutoff. Second, we impose an UV cutoff that corresponds to the Hubble scale at the transition between matter contraction and Ekpyrotic contraction. In other words, we integrate modes with value of $k$ smaller than $\mathcal{H}_{E}$. When considering particle production at the beginning of the bounce phase, we need to consider modes with $k$ between $\mathcal{H}_{E}$ and $\mathcal{H}_{B-}$. During the bounce phase, we take the Planck scale to be the UV cutoff, so we integrate modes with scale between $\mathcal{H}_{B-}$ and $a_{B} M_{p}$ (see Fig. 1 for a pictorial representation).

Using Eq. 46 for the number of particles created at $t_{E}$, performing the integral (47), and dividing the resulting energy density by the background energy density $\rho_{\text {back }} \simeq 3 M_{p}^{2} H_{E}^{2}$, we find the following result for the energy density of the particles produced during the matter 
phase of contraction (as a fraction of the background energy density):

$$
\begin{aligned}
\frac{\rho_{\chi}}{\rho_{\text {back }}} \simeq & \frac{m^{2}}{336 \pi^{2} M_{p}^{2} H_{E}^{2}}\left[30 m^{2}-7 q \frac{\left(\tilde{\eta}_{B-}+\eta_{E}\right)}{\left(\tilde{\eta}_{B-}-\eta_{E}\right)^{3}}\right] \\
& \times \ln \left(\frac{a_{B-} H_{B-}}{k_{\min }}\right),
\end{aligned}
$$

where $k_{\min }$ represents the comoving wave number of the fluctuating mode with the largest wavelength.

Let us explore the consequences of Eq. (48). Overall, the energy density of particle production after the matter phase of contraction is very small compared to the background energy density. The prefactor of Eq. (48) can be of order 1 , but the square bracket is suppressed due to the mass squared term and the second term is suppressed due to the large duration of the Ekpyrotic phase. The latter is necessary to dilute unwanted anisotropies (see Ref. 20]). Finally, the log term could be arbitrarily large for arbitrarily small values of $k_{\min }$, but recalling that the fluctuations must reenter the Hubble radius after the bounce, this term cannot contribute to the energy density significantly.

\section{Particle number at the beginning of the bounce phase}

Using the same technique as above, we require the mode solutions $\chi_{k}$ given by Eqs. (27) and (32) to be continuous at the transition time $t_{B-}$. The matching conditions then yield the following Bogoliubov coefficient:

$$
\beta_{k} \simeq e^{-i k\left(\eta_{B-}-\tilde{\eta}_{E}\right)-i \omega_{k}^{b} \eta_{B}-} \frac{\omega_{k}^{b}-k}{2 \sqrt{3 k \omega_{k}^{b}}},
$$

where we recall that the effective frequency in the bounce phase is given by Eq. (31). Up to leading order in $k$, we find the resulting number of particles to be given by

$$
n_{k}=\left|\beta_{k}\right|^{2} \simeq \frac{a_{B}^{4}\left(m^{2}-\Upsilon\right)^{2}}{48 k^{4}} .
$$

Using the fact that $m^{2} \ll \Upsilon$ and, since the bounce phase is short, assuming that $a\left(\eta_{B-}\right) \simeq a_{B}$, the energy density of particle production is found to be

$$
\rho_{\chi} \simeq \frac{\Upsilon^{2}}{96 \pi^{2}} \ln \left(\frac{a_{B-} H_{B-}}{a_{E} H_{E}}\right),
$$

and in terms of a fraction of the background energy density, we find

$$
\frac{\rho_{\chi}}{\rho_{\mathrm{back}}} \simeq \frac{1}{288 \pi^{2} M_{p}^{2} t_{B-}^{2}} \ln \left(\frac{a_{B-} H_{B-}}{a_{E} H_{E}}\right) .
$$

Note that we used the relation $H_{B-}=\Upsilon t_{B-}$.

This last result shows that gravitational particle production starts to become important in the Ekpyrotic phase of contraction. If the bounce phase is very fast (a few Planck times for instance), then the energy density of particle production is of the order of $\mathcal{O}\left(10^{-4}\right)$ times the background energy density. However, recent studies suggest that the bounce phase should last about $10^{3}-10^{4}$ Planck times to obtain the correct amplitude of cosmological perturbations and a sufficiently small tensor-toscalar ratio ([37, 38]), which reduces the fraction (52).

\section{Particle number at the end of the bounce phase}

For the transition in the equation of state occurring at $t_{B+}$, we require continuity of the mode solutions given by Eqs. (32) and (37). Using the same techniques as before, we find the Bogoliubov coefficient $\beta_{k}$ and the resulting number of particles. The latter is found to be

$$
n_{k} \simeq \frac{a_{B}^{4} \Upsilon^{2}}{48 k^{4}}\left(4 \Upsilon^{2} t_{B+}^{4}-\Upsilon t_{B+}^{2}-1\right)^{2},
$$

where we used the facts that $m^{2} \ll \Upsilon, a\left(\eta_{B+}\right) \simeq a_{B}$, and $\eta_{B+} \simeq t_{B+} / a_{B+}$. The resulting energy density in terms of the background energy density is found to be

$$
\frac{\rho_{\chi}}{\rho_{\text {back }}} \simeq \frac{\left(4 \Upsilon^{2} t_{B+}^{4}-\Upsilon t_{B+}^{2}-1\right)^{2}}{288 \pi^{2} M_{p}^{2} t_{B+}^{2}} \ln \left(\frac{a_{B} M_{p}}{a_{B-} H_{B-}}\right) .
$$

Here again, we see that particles have been produced. In fact, we see that Eqs. (52) and (54) share the same denominator if one assumes that the bounce phase is symmetric $\left(\left|t_{B-}\right|=\left|t_{B+}\right|\right)$. However, the energy density after the bounce phase has an extra factor which tends to 1 as the bounce duration tends to 0 . So, in the short bounce limit, the energy densities of particle production at the end of the Ekpyrotic phase and at the end of the bounce phase are comparable, whereas for a longer bounce, the contribution from the bounce phase dominates. At this point, the energy density of particle production is at most $\mathcal{O}\left(10^{-3}\right)$ times the background energy density.

\section{Reheating time and reheating temperature}

We found in the previous section that particles are gravitationally produced throughout the evolution of the Universe, but that the dominant contributions came from late in the Ekpyrotic phase of contraction and from the bounce phase. We also found that the energy density of these particles could reach about $10^{-3}$ times the background energy density at the end of the bounce phase. On one hand, this is not enough to disturb the background evolution, i.e., it does not lead to large backreaction effects. On the other hand, this is enough to reheat the Universe since after the bounce phase, particles that have been produced will redshift less fast than the background field and will hence ultimately become dominant.

Specifically, we know that $\rho_{\chi} \sim a^{-4}$, whereas $\rho_{\text {back }} \sim$ $a^{-6}$. Defining the "reheating time" $t_{R}$ as the time when 
the total energy density of particle production equals the background energy density,

$$
\rho_{\chi, \text { total }}\left(t_{R}\right)=\rho_{\text {back }}\left(t_{R}\right),
$$

we find that reheating occurs at

$$
t_{R}=\tilde{t}_{B+}+\frac{M_{p}^{3} a_{B+}^{6}}{\left(3 \rho_{*}\right)^{3 / 2}\left(t_{B+}-\tilde{t}_{B+}\right)^{2}},
$$

where

$$
\begin{aligned}
\rho_{*}= & \frac{\Upsilon^{2} a_{B+}^{4}}{96 \pi^{2}}\left[\ln \left(\frac{a_{B-} H_{B-}}{a_{E} H_{E}}\right)\right. \\
& \left.+\left(4 \Upsilon^{2} t_{B+}^{4}-\Upsilon t_{B+}^{2}-1\right)^{2} \ln \left(\frac{a_{B} M_{p}}{a_{B-} H_{B-}}\right)\right]
\end{aligned}
$$

is the sum of the energy densities from the Ekpyrotic phase and the bounce phase such that

$$
\rho_{\chi, \text { total }}\left(t_{B+}\right)=\rho_{*} a^{-4}\left(t_{B+}\right) .
$$

Finally, using the relation

$$
\rho_{\chi, \mathrm{total}}\left(t_{R}\right)=\frac{\pi^{2}}{15} T_{R}^{4}
$$

we find that the reheating temperature is given by

$$
\begin{aligned}
T_{R}= & \left(\frac{15}{9}\right)^{1 / 4} \frac{\rho_{*}^{3 / 4}}{\pi^{1 / 2} M_{p} H_{B+} a_{B+}^{3}} \\
= & \frac{10^{1 / 4}}{48 \pi^{2}} \frac{\Upsilon^{3 / 2}}{M_{p} H_{B+}}\left[\ln \left(\frac{a_{B-} H_{B-}}{a_{E} H_{E}}\right)\right. \\
& \left.+\left(4 \Upsilon^{2} t_{B+}^{4}-\Upsilon t_{B+}^{2}-1\right)^{2} \ln \left(\frac{a_{B} M_{p}}{a_{B-} H_{B-}}\right)\right]^{3 / 4} .
\end{aligned}
$$

For a short bounce, this temperature can be of the order of $\mathcal{O}\left(10^{-5}\right) M_{p}$, about an order of magnitude below the GUT scale. Using parameter values that better suit the recent observations ([37]), we find $T_{R} \sim \mathcal{O}\left(10^{-7}\right) M_{p}$.

Let us briefly compare reheating in inflationary cosmology and the bounce reheating mechanism by Parker particle production discussed here. Neither Parker particle production nor inflationary preheating produce a state with a thermal distribution of particles at the time that matter starts to dominate the energy density. A period of thermalization (e.g. via perturbative processes) is required both in inflation and in our bouncing cosmology. In fact, the processes involved will be identical. If we compare the energy density at which regular matter starts to dominate, then the value $T_{R} \sim \mathcal{O}\left(10^{-7}\right) M_{p}$ in our scenario is comparable to the "temperature" after preheating in intermediate energy inflation models. For a review of the challenges of the actual thermalization process the reader is referred to [1].

\section{BACKREACTION OF THERMAL PARTICLES FROM COSMIC FLUCTUATIONS}

In this section we take another view on the backreaction of the produced particles on the background dynamics. Instead of focusing directly on the number of produced particles and computing their associated energy density we will consider the energy-momentum tensor of the scalar field and directly compute its backreaction.

We know that for a scalar field $\psi$ with a canonical Lagrangian given by Eq. (8), the energy density due to modes with $k \gg \mathcal{H}$ is given by

$$
\rho_{\mathrm{br}} \simeq \frac{1}{2}\left\langle(\delta \dot{\psi})^{2}\right\rangle+\frac{1}{2 a^{2}}\left\langle(\nabla \delta \psi)^{2}\right\rangle+\frac{1}{2} m^{2}\left\langle(\delta \psi)^{2}\right\rangle,
$$

where $\delta \psi$ denotes the fluctuation of the scalar field. Note that in contrast to a field $\phi$ responsible for Ekpyrotic contraction, $\psi$ has no homogeneous nonvanishing background value. In the above expectation values, only subHubble Fourier modes are considered. If we wanted to discuss the backreaction of super-Hubble modes, it would be essential to include the metric fluctuations induced by the matter modes. In the context of inflationary cosmology, the backreaction formalism for long wavelength modes was developed in detail in Ref. [39].

Recalling that $\chi=a \psi$ and using the mode expansion of the $\chi$ field, it is straightforward to express the above energy density as

$$
\begin{aligned}
\rho_{\mathrm{br}} \simeq & \frac{1}{(2 \pi)^{3} a^{4}} \int d^{3} k \frac{1}{2}\left(\left|\chi_{k}^{\prime}\right|^{2}-\mathcal{H}\left(\chi_{k}^{\prime *} \chi_{k}+\chi_{k}^{\prime} \chi_{k}^{*}\right)\right. \\
& \left.+\left(\mathcal{H}^{2}+k^{2}+m^{2} a^{2}\right)\left|\chi_{k}\right|^{2}\right) .
\end{aligned}
$$

Expanding the $\chi_{k}$ modes as sums of positive and negative frequency modes [see Eq. [39] ] and using the fact that $\omega_{k} \simeq k$ in the large $k$ limit, the backreaction energy density becomes

$$
\rho_{\mathrm{br}} \simeq \frac{1}{(2 \pi)^{3} a^{4}} \int d^{3} k \frac{1}{2} \omega_{k}\left(\left|\alpha_{k}\right|^{2}+\left|\beta_{k}\right|^{2}\right) .
$$

Noting that $\left|\alpha_{k}\right|^{2}-\left|\beta_{k}\right|^{2}=1$ and $n_{k}=\left|\beta_{k}\right|^{2}$, we can reexpress the last result as

$$
\rho_{\mathrm{br}} \simeq \frac{1}{(2 \pi)^{3} a^{4}} \int d^{3} k \frac{1}{2} \omega_{k}+\frac{1}{(2 \pi)^{3} a^{4}} \int d^{3} k \omega_{k} n_{k} .
$$

The first term of this equation is the (divergent) vacuum energy, which we ignore. The second term is what we wished to show as being the energy density of particle production and it is what we used throughout Sec. IIIB.

\section{PARTICLE PRODUCTION THROUGH DIRECT INTERACTIONS}

In inflationary cosmology particle production through direct interactions between the matter field and the inflaton field is much more important than gravitational 
particle production. The energy density due to gravitational particle production is of the order $H^{4}$, where $H$ is the Hubble expansion rate during inflation, whereas the energy density transferred to matter via preheating is proportional to $H^{2} M_{p}^{2}$ which is parametrically larger by a factor of $\left(M_{p} / H\right)^{2}$. Thus, an obvious question to ask is how large the contribution of direct interactions to matter production is in our matter bounce scenario.

The efficiency of the parametric resonance instability [2] underlying preheating can be traced [5] to the fact that the inflaton oscillates many times through $\varphi=0$. During each crossing, the adiabaticity condition for the matter modes is violated, leading to many bursts of particle production. In contrast, in our model $\phi$ crosses zero only once, and so less particle production through direct interactions is expected.

On the other hand, in the emergent Galileon cosmology of [8], particle production via direct interactions turns out to be very efficient in spite of the fact that the dynamical background field crosses $\phi=0$ only once [9]. However, as explained in [9], this is unexpected and due to the particular couplings of the model. Hence, taking the lessons of 9 into account we expect a small amount of particle production via interactions. In the following, we will verify this expectation.

To obtain direct particle production we need to add an interaction Lagrangian to the problem (as one needs to in order to obtain inflationary reheating). We take this coupling to be given by the last term in the following Lagrangian

$$
\mathcal{L}=K(\phi, X)+G(\phi, X) \square \phi+P(\psi, Y)-\frac{1}{2} \lambda^{2} \phi^{2} \psi^{2} M_{p}^{2},
$$

for some real coupling constant $\lambda$. We assume that the coupling only takes place during the bounce phase where we expect particle production to be most significant. Outside the bounce phase, we assume that the coupling constant $\lambda$ is very small.

Using the above Lagrangian with the interaction term, the equation of motion (EoM) for $\psi$ in Fourier space becomes

$$
\ddot{\psi}_{k}+3 H \dot{\psi}_{k}+\left(\frac{k^{2}}{a^{2}}+m^{2}+\lambda^{2} \phi^{2} M_{p}^{2}\right) \psi_{k}=0 .
$$

Defining a new auxiliary field as $X_{k}(t) \equiv a^{3 / 2}(t) \psi_{k}(t)$, the equation of motion simplifies and becomes

$$
\ddot{X}_{k}+\omega_{k}^{2} X_{k}=0
$$

where the time-dependent effective frequency is given by

$$
\omega_{k}^{2}=\frac{k^{2}}{a^{2}}+m^{2}+\lambda^{2} \phi^{2} M_{p}^{2}-\frac{9}{4} H^{2}-\frac{3}{2} \dot{H} .
$$

Since the background evolution in the bounce phase is well known (see 20]), we could write down the form of Eq. 68 explicitly, but we will be mainly interested in the effective frequency close to the bounce point where $\phi$ crosses 0 . We note that

$$
\phi(t) \simeq \frac{\sqrt{\pi}}{2} \dot{\phi}_{B} T \operatorname{erf}\left(\frac{t}{T}\right)
$$

where $T$ is given by

$$
T \simeq \frac{H_{B+}}{\Upsilon} \sqrt{\frac{2}{\ln \left(\dot{\phi}_{B}^{2} / 6 H_{B+}^{2}\right)}}
$$

Thus, as long as the ratio $t / T$ remains small, it is sufficient to expend the effective frequency squared to second order in time as follows,

$$
\begin{aligned}
\omega_{k}^{2}(t) \simeq & \left(\frac{k^{2}}{a_{B}^{2}}+m^{2}-\frac{3 \Upsilon}{2}\right) \\
& +\left(\lambda^{2} \dot{\phi}_{B}^{2} M_{p}^{2}-\frac{9 \Upsilon^{2}}{4}-\frac{k^{2} \Upsilon}{a_{B}^{2}}\right) t^{2} \\
\equiv & b+c t^{2},
\end{aligned}
$$

where we have denoted the zeroth-order term as $b$ and the coefficient of the quadratic term as $c$.

To determine when particle production occurs, we need to find when the adiabaticity condition is violated. This is the case when

$$
\left|\dot{\omega}_{k}\right| \gtrsim \omega_{k}^{2}
$$

Using Eq. (71) for the effective frequency, the inequality (72) is equivalent to

$$
c^{3} t^{6}+3 b c^{2} t^{4}+c\left(3 b^{2}-c\right) t^{2}+b^{3} \lesssim 0
$$

Since the constant term in the above equation is negligible when $\lambda$ is large, we can solve for $t$ and thus we find that particle production occurs without interruption for $t_{i} \lesssim t \lesssim t_{f}$ with

$$
t_{f}=\sqrt{\frac{\sqrt{4 c-3 b^{2}}-3 b}{2 c}}
$$

and $t_{i}=-t_{f}$. We note that this is only valid if $c>3 b^{2}$, which translates into an upper bound ${ }^{1}$,

$$
k_{\max }=a_{B} \sqrt{\frac{4 \Upsilon+\sqrt{3 \lambda^{2} \dot{\phi}_{B}^{2} M_{p}^{2}-11 \Upsilon^{2}}}{3}},
$$

as long as $\lambda>3 \Upsilon / \dot{\phi}_{B} M_{p}$.

In order to solve the EoM, let us write down the solution in the form of

$$
\begin{aligned}
X_{k}(t)= & \frac{1}{\sqrt{2 \omega_{k}(t)}} \\
& \times\left[\alpha_{k}(t) e^{-i \int_{t_{i}}^{t} d \tilde{t} \omega_{k}(\tilde{t})}+\beta_{k}(t) e^{i \int_{t_{i}}^{t} d \tilde{t} \omega_{k}(\tilde{t})}\right] .
\end{aligned}
$$

${ }^{1}$ As in Sec. III B we assume $m^{2} \ll \Upsilon$. 
Its conjugate momentum is then given by

$$
\begin{aligned}
\Pi_{k}(t)= & i \sqrt{\frac{\omega_{k}(t)}{2}} \\
& \times\left[-\alpha_{k}(t) e^{-i \int_{t_{i}}^{t} d \tilde{t} \omega_{k}(\tilde{t})}+\beta_{k}(t) e^{i \int_{t_{i}}^{t} d \tilde{t} \omega_{k}(\tilde{t})}\right],
\end{aligned}
$$

and the Bogoliubov coefficients satisfy the following equations:

$$
\begin{aligned}
\dot{\alpha}_{k} & =\frac{\dot{\omega}_{k}}{2 \omega_{k}} e^{2 i \int_{t_{i}}^{t} d \tilde{t} \omega_{k}(\tilde{t})} \beta_{k}, \\
\dot{\beta}_{k} & =\frac{\dot{\omega}_{k}}{2 \omega_{k}} e^{-2 i \int_{t_{i}}^{t} d \tilde{t} \omega_{k}(\tilde{t})} \alpha_{k} .
\end{aligned}
$$

To set the initial conditions, we require that there are no particles at $t_{i}$, which implies that $\alpha_{k}\left(t_{i}\right)=1$ and $\beta_{k}\left(t_{i}\right)=0$.

Once we find the solution $X_{k}(t)$, we know that the total number of particles that will have been produced is given by

$$
n_{k}\left(t_{f}\right)=\frac{\omega_{k}\left(t_{f}\right)}{2}\left(\frac{\left|\dot{X}_{k}\left(t_{f}\right)\right|^{2}}{\omega_{k}^{2}\left(t_{f}\right)}+\left|X_{k}\left(t_{f}\right)\right|^{2}\right)-\frac{1}{2},
$$

or equivalently by

$$
n_{k}\left(t_{f}\right)=\left|\beta_{k}\left(t_{f}\right)\right|^{2} .
$$

An approximate solution is given by (see the Appendix for details)

$$
n_{k}\left(t_{f}\right) \simeq \sinh ^{2}\left[\frac{t_{f}^{2} \sqrt{c}}{2}-\frac{b}{2 \sqrt{c}} \ln \left(1+\frac{c t_{f}^{2}}{b}\right)\right] .
$$

The energy density of particle production at the end of the bounce phase is then given by

$$
\rho_{X}\left(t_{B+}\right)=\frac{1}{2 \pi^{2} a\left(t_{B+}\right)^{4}} \int_{0}^{k_{\max }} d k k^{2} \omega_{k}\left(t_{B+}\right) n_{k}\left(t_{f}\right),
$$

where we used the fact that $n_{k}\left(t_{B+}\right)=n_{k}\left(t_{f}\right)$ since particle production stops before the end of the bounce phase. As explained in the Appendix, the result for $n_{k}\left(t_{f}\right)$ is only valid for $b>0$, which imposes a lower bound $k_{\min }=a_{B} \sqrt{3 \Upsilon / 2}$. Since we are interested in the limit where $\lambda \gg \Upsilon$, we notice that $k_{\max } \gg k_{\min }$ meaning that the integral is dominated by its UV cutoff. Thus, we can effectively take the lower integration bound to be 0 .

The integrand of Eq. (83) is a complicated function of $k$, but it is straightforward to find an upper bound to the overall contribution. For large values of the coupling constant, we see from Eq. (68) that the effective frequency squared at the end of the bounce phase is dominated by the term $\lambda^{2} \phi\left(t_{B+}\right)^{2} M_{p}^{2}$. We also notice that $n_{k}\left(t_{f}\right) \lesssim \sinh ^{2}(1 / 2)$ for all allowed values of $k$, so Eq. (83) simplifies to become the inequality

$$
\rho_{X}\left(t_{B+}\right) \lesssim \frac{a_{B}^{3}\left(\lambda \dot{\phi}_{B} M_{p}\right)^{5 / 2} H_{B+} \operatorname{erf}\left(t_{B+} / T\right) \sinh ^{2}(1 / 2)}{3^{7 / 4}(2 \pi)^{3 / 2} a_{B+}^{4} \Upsilon \sqrt{\ln \left(\dot{\phi}_{B}^{2} / 6 H_{B+}^{2}\right)}} .
$$

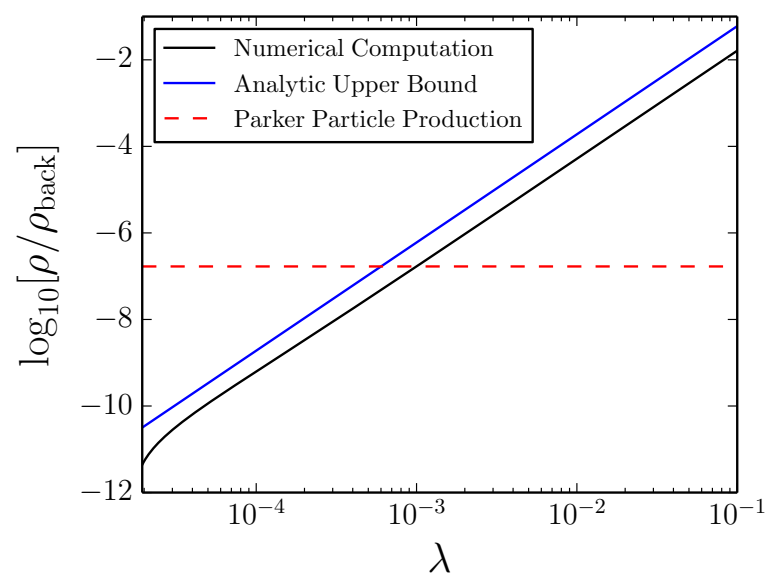

FIG. 2: Plot of the ratio of the energy density of particle production to the background energy density as a function of the coupling constant. The slope of the Hubble parameter and the physical duration of the bounce are taken to be $\Upsilon=$ $5.5 \times 10^{-7} M_{p}^{2}$ and $t_{B+}=2.1 \times 10^{3} M_{p}^{-1}$, respectively. The blue curve shows the analytic upper bound found in Eq. (84) and the black curve was obtained by solving the EoM and the integral for the energy density numerically. The dashed red line is the contribution from Parker particle production that we found earlier [see Eq. [54].

This can be compared to the energy density of Parker particle production (recall Eq. (54)),

$$
\begin{aligned}
\frac{\rho_{X}\left(t_{B+}\right)}{\rho_{\chi}\left(t_{B+}\right)} \lesssim & \frac{8 \sqrt{2 \pi} a_{B}^{3}\left(\lambda \dot{\phi}_{B} M_{p}\right)^{5 / 2} t_{B+} \operatorname{erf}\left(\frac{t_{B+}}{T}\right) \sinh ^{2} \frac{1}{2}}{3^{3 / 4} a_{B+}^{4} \Upsilon^{2} \ln \left(\frac{a_{B} M_{p}}{a_{B-} H_{B-}}\right) \sqrt{\ln \left(\frac{\dot{\phi}_{B}^{2}}{6 H_{B+}^{2}}\right)}} \\
& \times\left(4 \Upsilon^{2} t_{B+}^{4}-\Upsilon t_{B+}-1\right)^{-2} .
\end{aligned}
$$

From this result, we see that for a fixed energy scale $H_{B+}$ and a fixed coupling constant $\lambda$, the ratio of the energy densities tends to zero as $\Upsilon \rightarrow 0$ or as $\Upsilon \rightarrow \infty$. This means that in the limit where the bounce is either infinitely long or infinitely short, the energy density from Parker particle production will always dominate the energy density from interaction. However, for typical bounce durations, the ratio of the energy densities depends on the size of the coupling constant. An example is shown in Fig. 2 where we see that particle production from interaction can contribute significantly to Parker particle production, but only if the coupling between the two fields is quite large. In general, for smaller values of the coupling constant, the energy density from Parker particle production dominates over the energy density from interaction.

\section{CONCLUSIONS}

We have considered Parker particle production in the two-field matter bounce model of [19], a prototypical ex- 
ample of a nonsingular matter bounce which can generate a scale-invariant spectrum of cosmological perturbations and which is stable against anisotropies near the bounce point. The stability to anisotropies is obtained via an Ekpyrotic phase of contraction. This phase of Ekpyrosis, on the other hand, also washes out any matter and radiation which might have existed early in the contracting phase. Hence, to make the model viable, a reheating mechanism is required.

We have shown that in our background, gravitational Parker particle production is sufficiently effective to reheat the Universe to high temperatures. For minimal coupling between the scalar field $\phi$ yielding the Ekpyrotic contraction and the nonsingular bounce and regular matter the effect of particle production through interactions is generally small, but could contribute to Parker particle production if the coupling were large. We thus see that we do not need to introduce extra ingredients into our matter bounce model in order to obtain a hot postbounce universe dominated by regular matter and radiation. This contrast with the case of inflationary cosmology, where the direct coupling of the inflaton field with matter generically produces an energy density in the reheat fields which is of the order of the inflationary energy density, whereas the contribution of Parker particle production is of the order $\rho \sim H^{4}$ and which is hence suppressed compared to the density of particles produced by preheating by a factor of order $\left(H / M_{p}\right)^{2}$. The reason for this difference is related to the fact that in the case of inflation the inflaton field loses most of its energy density during the reheating process, whereas in our nonsingular bounce the field $\phi$ retains most of its energy.

Finally, we note that the methods developed here are applicable to the study of Parker particle production in other nonsingular bouncing models, e.g. in the new Ekpyrotic universe [40]. Based on the arguments of the previous paragraph we conjecture that also in these other models, Parker particle production will be sufficient to reheat the Universe, and that Parker particle production will not be suppressed relative to particle production via direct couplings. To support this conjecture, we point out that Parker particle production has been shown to be efficient for certain models of the matter bounce in loop quantum cosmology 41.

\section{Acknowledgments}

J. Q. acknowledges the Natural Sciences and Engineering Research Council (NSERC) of Canada for financial support under a CGS M scholarship. The research of R. B. and Y. C. is supported by an NSERC Discovery grant and by funds from the Canada Research Chair program.

\section{Appendix A: Solving for the Bogoliubov coefficients in the bounce phase}

We want to solve the set of coupled first-order ordinary differential equations

$$
\begin{gathered}
\dot{\alpha}_{k}=\frac{\dot{\omega}_{k}}{2 \omega_{k}} e^{2 i \int_{t_{i}}^{t} d \tilde{t} \omega_{k}(\tilde{t})} \beta_{k}, \\
\dot{\beta}_{k}=\frac{\dot{\omega}_{k}}{2 \omega_{k}} e^{-2 i \int_{t_{i}}^{t} d \tilde{t} \omega_{k}(\tilde{t})} \alpha_{k}
\end{gathered}
$$

To simplify the treatment, let us define

$$
g_{k}^{ \pm}(t) \equiv \frac{\dot{\omega}_{k}}{2 \omega_{k}} e^{ \pm 2 i \int_{t_{i}}^{t} d \tilde{t} \omega_{k}(\tilde{t})},
$$

together with the matrix

$$
A_{k}(t) \equiv\left(\begin{array}{cc}
0 & g_{k}^{+}(t) \\
g_{k}^{-}(t) & 0
\end{array}\right)
$$

and the vector

$$
\mathbf{y}_{k}(t) \equiv\left(\begin{array}{c}
\alpha_{k}(t) \\
\beta_{k}(t)
\end{array}\right)
$$

Then, the system to solve simply reads

$$
\dot{\mathbf{y}}_{k}(t)=A_{k}(t) \mathbf{y}_{k}(t)
$$

and the initial conditions $\alpha_{k}\left(t_{i}\right)=1$ and $\beta_{k}\left(t_{i}\right)=0$ can be written as

$$
\mathbf{y}_{k}\left(t_{i}\right)=\left(\begin{array}{l}
1 \\
0
\end{array}\right) \equiv \mathbf{y}_{0} .
$$

We make use of the Magnus expansion [42, which tells that the solution to the above initial value problem is given by

$$
\mathbf{y}_{k}(t)=e^{\Omega_{k, 1}(t)+\Omega_{k, 2}(t)+\cdots} \mathbf{y}_{0}
$$

where

$$
\begin{aligned}
& \Omega_{k, 1}(t)=\int_{t_{i}}^{t} d t_{1} A_{k}\left(t_{1}\right) \\
& \Omega_{k, 2}(t)=\frac{1}{2} \int_{t_{i}}^{t} d t_{1} \int_{t_{i}}^{t_{1}} d t_{2}\left[A_{k}\left(t_{1}\right), A_{k}\left(t_{2}\right)\right],
\end{aligned}
$$

and so on. Here, we consider the first-order approximation only. Defining

$$
I_{k}^{ \pm}(t) \equiv \int_{t_{i}}^{t} d t_{1} g_{k}^{ \pm}\left(t_{1}\right)
$$

the solution becomes

$$
\mathbf{y}_{k}(t) \simeq \exp \left(\begin{array}{cc}
0 & I_{k}^{+}(t) \\
I_{k}^{-}(t) & 0
\end{array}\right) \mathbf{y}_{0}
$$


and therefore the Bogoliubov coefficients are given by

$$
\begin{aligned}
& \alpha_{k}(t) \simeq \cosh \sqrt{I_{k}^{+}(t) I_{k}^{-}(t)} \\
& \beta_{k}(t) \simeq \sqrt{\frac{I_{k}^{-}(t)}{I_{k}^{+}(t)}} \sinh \sqrt{I_{k}^{+}(t) I_{k}^{-}(t)} .
\end{aligned}
$$

In the present case for $\omega_{k}(t) \simeq b+c t^{2}$, the function $g_{k}^{ \pm}(t)$ defined in Eq. A3 can be evaluated exactly. Then, under the large- $\lambda$ assumption and as long as $b>0$, the integral defined in Eq. A11 evaluated at the end of the particle production phase becomes

$$
I_{k}^{ \pm}\left(t_{f}\right) \simeq \mp \frac{i e^{ \pm i t_{f} \sqrt{b+c t_{f}^{2}}}}{2 \sqrt{c}}\left[c t_{f}^{2}-b \ln \left(1+\frac{c t_{f}^{2}}{b}\right)\right] .
$$

Therefore, the number of particles that has been produced is found to be

$$
n_{k}\left(t_{f}\right)=\left|\beta_{k}\left(t_{f}\right)\right|^{2} \simeq \sinh ^{2} \frac{1}{2 \sqrt{c}}\left[c t_{f}^{2}-b \ln \left(1+\frac{c t_{f}^{2}}{b}\right)\right] .
$$

[1] R. Allahverdi, R. Brandenberger, F. -Y. Cyr-Racine and A. Mazumdar, Ann. Rev. Nucl. Part. Sci. 60, 27 (2010) arXiv:1001.2600 [hep-th]].

[2] J. H. Traschen and R. H. Brandenberger, Phys. Rev. D 42, 2491 (1990)

[3] L. Kofman, A. D. Linde and A. A. Starobinsky, Phys. Rev. Lett. 73, 3195 (1994) hep-th/9405187.

[4] Y. Shtanov, J. H. Traschen and R. H. Brandenberger, Phys. Rev. D 51, 5438 (1995) hep-ph/9407247.

[5] L. Kofman, A. D. Linde and A. A. Starobinsky, Phys. Rev. D 56, 3258 (1997) hep-ph/9704452.

[6] R. H. Brandenberger and C. Vafa, Nucl. Phys. B 316, 391 (1989);

A. Nayeri, R. H. Brandenberger and C. Vafa, Phys. Rev. Lett. 97, 021302 (2006) hep-th/0511140.

[7] R. H. Brandenberger, String Cosmology, J.Erdmenger (Editor). Wiley, 2009. p.193-230 arXiv:0808.0746 [hepth]];

T. Battefeld and S. Watson, Rev. Mod. Phys. 78, 435 (2006) hep-th/0510022.

[8] P. Creminelli, A. Nicolis and E. Trincherini, JCAP 1011, 021 (2010) arXiv:1007.0027 [hep-th]].

[9] L. Perreault Levasseur, R. Brandenberger and A. -C. Davis, Phys. Rev. D 84, 103512 (2011) arXiv:1105.5649 [astro-ph.CO]].

[10] D. Wands, Phys. Rev. D 60, 023507 (1999) arXiv:grqc/9809062.

[11] F. Finelli and R. Brandenberger, Phys. Rev. D 65, 103522 (2002) arXiv:hep-th/0112249.

[12] Y. F. Cai, T. Qiu, Y. S. Piao, M. Li and X. Zhang, JHEP 0710, 071 (2007) arXiv:0704.1090 [gr-qc]].

[13] C. Lin, R. H. Brandenberger and L. Perreault Levasseur, JCAP 1104, 019 (2011) arXiv:1007.2654 [hep-th]].

[14] C. Kounnas, H. Partouche and N. Toumbas, Nucl. Phys. B 855, 280 (2012) arXiv:1106.0946 [hep-th]];

I. Florakis, C. Kounnas, H. Partouche and N. Toumbas, Nucl. Phys. B 844, 89 (2011) arXiv:1008.5129 [hep-th]]; R. H. Brandenberger, C. Kounnas, H. Partouche, S. P. Patil and N. Toumbas, JCAP 1403, 015 (2014) arXiv:1312.2524 [hep-th]].

[15] R. Brandenberger, Phys. Rev. D 80, 043516 (2009) arXiv:0904.2835 [hep-th]].

[16] T. Biswas, A. Mazumdar and W. Siegel, JCAP 0603, 009 (2006) arXiv:hep-th/0508194.

[17] E. Wilson-Ewing, JCAP 1303, $026 \quad$ (2013) arXiv:1211.6269 [gr-qc]];
Y. -F. Cai and E. Wilson-Ewing, JCAP 1403, 026 (2014) arXiv:1402.3009 [gr-qc]].

[18] V. A. Belinsky, I. M. Khalatnikov and E. M. Lifshitz, Adv. Phys. 19, 525 (1970).

[19] Y. -F. Cai, D. A. Easson and R. Brandenberger, JCAP 1208, 020 (2012) arXiv:1206.2382 [hep-th]].

[20] Y. -F. Cai, E. McDonough, F. Duplessis and R. H. Brandenberger, JCAP 1310, 024 (2013) arXiv:1305.5259 [hep-th]].

[21] Y. -F. Cai, R. Brandenberger and P. Peter, Class. Quant. Grav. 30, 075019 (2013) arXiv:1301.4703 [gr-qc]].

[22] Y. -F. Cai, Sci. China Phys. Mech. Astron. 57, 1414 (2014) arXiv:1405.1369 [hep-th]].

[23] L. Parker, Phys. Rev. Lett. 21, 562 (1968).

[24] L. Parker, Phys. Rev. 183, 1057 (1969).

[25] L. Parker, Phys. Rev. D 3, 346 (1971) [Erratum-ibid. D 3, 2546 (1971)].

[26] C. Armendariz-Picon, V. F. Mukhanov and P. J. Steinhardt, Phys. Rev. D 63, 103510 (2001) astro$\mathrm{ph} / 0006373$.

[27] G. W. Horndeski, Int. J. Theor. Phys. 10, 363 (1974); A. Nicolis, R. Rattazzi and E. Trincherini, Phys. Rev. D 79, 064036 (2009) arXiv:0811.2197 [hep-th]].

[28] J. Khoury, B. A. Ovrut, P. J. Steinhardt and N. Turok, Phys. Rev. D 64, 123522 (2001) arXiv:hep-th/0103239; J. Khoury, B. A. Ovrut, N. Seiberg, P. J. Steinhardt and N. Turok, Phys. Rev. D 65, 086007 (2002) arXiv:hepth/0108187.

[29] J. K. Erickson, D. H. Wesley, P. J. Steinhardt and N. Turok, Phys. Rev. D 69, 063514 (2004) hepth/0312009.

[30] N. Arkani-Hamed, H. -C. Cheng, M. A. Luty and S. Mukohyama, JHEP 0405, 074 (2004) hepth/0312099.

[31] A. D. Dolgov and A. D. Linde, Phys. Lett. B 116, 329 (1982).

[32] L. F. Abbott, E. Farhi and M. B. Wise, Phys. Lett. B 117, 29 (1982).

[33] A. D. Dolgov and D. P. Kirilova, Sov. J. Nucl. Phys. 51, 172 (1990) [Yad. Fiz. 51, 273 (1990)].

[34] Y. -F. Cai, R. Brandenberger and X. Zhang, Phys. Lett. B 703, 25 (2011) arXiv:1105.4286 [hep-th]].

[35] P. Peter and N. Pinto-Neto, Phys. Rev. D 66, 063509 (2002) hep-th/0203013;

P. Peter, N. Pinto-Neto and D. A. Gonzalez, JCAP 0312, 003 (2003) hep-th/0306005; 
J. Martin and P. Peter, Phys. Rev. D 68, 103517 (2003) hep-th/0307077;

S. Alexander, T. Biswas and R. H. Brandenberger, arXiv:0707.4679 [hep-th];

R. Brandenberger, H. Firouzjahi and O. Saremi, JCAP 0711, 028 (2007) arXiv:0707.4181 [hep-th]];

Y. F. Cai, T. Qiu, R. Brandenberger, Y. S. Piao and X. Zhang, JCAP 0803, 013 (2008) arXiv:0711.2187[hepth]];

Y. -F. Cai, T. -t. Qiu, R. Brandenberger and X. -m. Zhang, Phys. Rev. D 80, 023511 (2009) arXiv:0810.4677 [hep-th]];

X. Gao, Y. Wang, W. Xue and R. Brandenberger, JCAP 1002, 020 (2010) arXiv:0911.3196 [hep-th]];

F. Finelli, P. Peter and N. Pinto-Neto, Phys. Rev. D 77, 103508 (2008) arXiv:0709.3074 [gr-qc]].

[36] N. Birrell and P. Davies, Quantum Fields in Curved Space (Cambridge Univ. Press, Cambridge, 1982).

[37] Y. F. Cai, J. Quintin, E. N. Saridakis and E. WilsonEwing, JCAP 1407, 033 (2014) arXiv:1404.4364 [astro-
ph.CO]].

[38] L. Battarra, M. Koehn, J. L. Lehners and B. A. Ovrut, JCAP 1407, 007 (2014) arXiv:1404.5067 [hep-th]].

[39] L. R. W. Abramo, R. H. Brandenberger and V. F. Mukhanov, Phys. Rev. D 56, 3248 (1997) grqc/9704037.

[40] A. Notari and A. Riotto, Nucl. Phys. B 644, 371 (2002) hep-th/0205019;

F. Finelli, Phys. Lett. B 545, 1 (2002) hep-th/0206112; E. I. Buchbinder, J. Khoury and B. A. Ovrut, Phys. Rev. D 76, 123503 (2007) hep-th/0702154;

P. Creminelli and L. Senatore, JCAP 0711, 010 (2007) hep-th/0702165;

J. -L. Lehners, P. McFadden, N. Turok and P. J. Steinhardt, Phys. Rev. D 76, 103501 (2007) hep-th/0702153 [HEP-TH]].

[41] J. Haro and J. Amorós, arXiv:1406.0369 [gr-qc].

[42] W. Magnus, Commun. Pure Appl. Math. 7, 649 (1954). 